\title{
Thermal effects of laser irradiation on maize seeds
}

\author{
Claudia Hernández Aguilar ${ }^{*}$, Flavio Arturo Domínguez Pacheco ${ }^{1}$, Alfredo Cruz Orea ${ }^{2}$, \\ and Rumen Ivanov Tsonchev ${ }^{3}$ \\ ${ }^{1}$ National Polytechnic Institute, Sepi-Esime, Zacatenco. Professional Unit ‘Adolfo López Mateos', Col. Lindavista, \\ México D.F., C.P. 07738, Mexico \\ ${ }^{2}$ Department of Physics, CINVESTAV - IPN, A.P. 14-740, Mexico D.F., C.P. 07360, Mexico \\ ${ }^{3}$ Doctorate in Engineering Sciences, Autonomy University of Zacatecas, A.P. 580, Zacatecas, Mexico
}

Received September 11, 2014; accepted April 1, 2015

\begin{abstract}
A b s t r a c t. It is important to know the temperature changes in seeds that have been irradiated with laser light because this could have substantial practical and theoretical importance. Thus, the thermal effects of low intensity laser irradiation on seeds was studied, showing variation of temperature produced by laser light applied during $60 \mathrm{~s}$ on two maize seed genotypes, 'Toluqueño' and 'Cacahuazintle': crystalline and floury, respectively, under two different conditions: natural colour and dyed black, evaluating the temperature changes by a thermal camera. The optical absorption spectra and the non-radiative relaxation time of the seeds were obtained using photoacoustic spectroscopy. The results indicate that it is possible to produce temperature changes, detected by an infrared camera, in crystalline and floury seeds when they are irradiated with a laser beam at a $650 \mathrm{~nm}$ wavelength and $27.4 \mathrm{~mW}$ power. The highest variation of temperature in the seeds was obtained for the black-dyed condition, these variations being 5.56 and $9.28^{\circ} \mathrm{C}$ for crystalline and floury seeds, respectively. Among the seeds, in the dyed condition, the floury seed had the lower non-radiative relaxation time, the higher optical absorption coefficient and a lower optical penetration length at the laser wavelength $(650 \mathrm{~nm})$.

$\mathrm{K}$ e y w o r d s: maize, temperature, thermal camera, laser
\end{abstract}

\section{INTRODUCTION}

Stimulation by low-intensity laser irradiation (LILI) in pre-sowing on agricultural seeds has been confirmed in numerous studies using cereal seeds, vegetables etc. (A ElKereti et al., 2013; Javed et al., 2011; Jia et al., 2013; Perveen et al., 2010, 2011; Srećković et al., 2014). The synergies of different events that occur when laser light interacts with seeds are currently under study, but the mechanism of the action of light on seeds is not suf-

*Corresponding author e-mail: clauhaj@yahoo.com ficiently clear (Zhang et al., 2011). In general, there are several hypotheses concerning the possible mechanisms of biological stimulation in seeds by the action of LILI, which assume that seeds contain molecules with very narrow optical absorption bands. A selective excitation of the chromophores of these molecules initiates some biochemical reactions (Popov et al., 2007). In the pre-sowing seed stimulation by LILI, the phytochromes, among other molecules, are excited by the absorption of the laser beam (Abdelgaphar and Tuleukhanov, 2013; Gao et al., 2014; Hernández et al., 2010; Muthusamy et al., 2012) and then the energy of the excited molecules is transformed into chemical energy for their subsequent growth processes (Jamil et al., 2013). In this way, the laser irradiation could break the kinetic equilibrium of seed germination and increase the internal energy of seeds (Ferdosizadeh et al., 2013).

Another hypothesis to be considered is the thermal action, which assumes that the acting factor of LILI is local heating of cells or cell elements that efficiently absorb light at the laser irradiation wavelength (Popov et al., 2007), ie a fraction of the excitation energy is converted to heat (Rassam et al., 2010). In this way, laser irradiation could cause enhancement of enzyme activities (Rassam et al., 2010; Wu et al., 2007; Zhang et al., 2011), which accelerates enzyme-mediated reactions through the electromagnetic field and heat energy affecting molecules in the cell (Chen et al., 2005a). Therefore, some authors agree that the effects of the interaction of the laser light with a biological 
system involve at least light, electromagnetic, and temperature effects (Abdelgaphar and Tuleukhanov, 2013; Chen et al., 2005a; 2005b).

Each event that takes place in a living system is linked with production or absorption of heat, which leads to temperature changes in the system. Therefore, the pre-sowing seed treatment by laser irradiation is also related to temperature changes (Jamil et al., 2013), which are supposed to be small (Abdelgaphar and Tuleukhanov, 2013). However, it is important to know the temperature variation in the seeds that have been irradiated with a laser beam because this could have substantial practical and theoretical importance. The variations of temperature will be a function of the seed characteristics, as well as the exposure time to the laser beam (Hernandez et al., 2010). The exposure times to red laser irradiation (wavelength from 618 to $780 \mathrm{~nm}$ ) have ranged from milliseconds, seconds, minutes to hours using several lasers (Aladjadjiyan, 2012; Hoseini et al., 2013; Khalifa and Ghandoor, 2011; Metwally et al., 2013; Mohammadi et al., 2012; Podleśny et al., 2012). Therefore, the objective of the present study was to determine the evolution of the temperature in maize seeds (Zea mays L.) when LILI was applied during $60 \mathrm{~s}$ and after $60 \mathrm{~s}$ of laser off, evaluating the temperature variation by using a thermal camera. Untreated seeds (I) and black-dyed seeds (II) were used in this investigation for two maize seed genotypes ('Cacahuazintle' and 'Toluqueño': floury and crystalline, respectively). In addition, measurements were performed by photoacoustic spectroscopy (PAS) to determine the optical absorption coefficient $(\beta)$ and the non-radiative relaxation time for the studied maize seeds, under the hypothesis that LILI produces temperature variations in maize seeds, which can be detected by an infrared camera, where these thermal changes vary depending on the seed optical absorption coefficient. At a higher seed optical absorption coefficient, higher warming at the seed surface is obtained.

Some authors have found that it is possible to increase the effect of laser irradiation by the use of photosensitizers as artificial dyes (Ouf and Abdel-Hady, 1999); in this sense, the present study is focused on identifying thermal changes that occur in the untreated and dyed seeds when they are irradiated with laser light. The seed industry uses agrochemicals, such as: artificial dyes, fungicides, fertilizers, etc. Thus, an increase of the knowledge concerning the explanation of mechanism of laser light effect on crop seeds; this could have practical relevance for a future application of the methods of laser stimulation in the agricultural sector of developing countries and prevent damage to the environment and human health.

\section{MATERIALS AND METHODS}

In the present research, two varieties of maize seeds were studied, which were provided by the Mexican Institute of Genetic for the Seed Quality Control. The studied seed varieties were 'Toluqueño' and 'Cacahuazintle': crystalline $\left(V_{1 C}\right)$ and floury $\left(V_{2 F}\right)$, respectively, which were grown during the agricultural cycle spring-summer 2011 in Valles Altos, Mexico, and prior to the study the seed lot was standardized in size and colour. The thicknesses of six seeds of each variety were measured by using a Vernier instrument and their average values were $3.78 \pm 0.21$ and $4.13 \pm 0.18 \mathrm{~mm}$ for the $V_{1 C}$ and $V_{2 F}$ varieties, respectively. The seed varieties had different pigmentation (different shade of white). Seeds of each variety were randomly selected to determine their optical absorption spectra and the non-radiative relaxation time of the seeds using photoacoustic spectroscopy (PAS). Subsequently, twelve seeds were selected from each variety: crystalline and floury. Six seeds of each variety were dyed black $\left(V_{1 C D}\right.$ and $\left.V_{2 F D}\right)$ and six seeds were in their natural colour $\left(V_{1 C N}\right.$ and $\left.V_{2 F N}\right)$ in order to identify temperature changes from the obtained thermal images of each variety of seeds in the two conditions, natural $(\mathrm{N})$ and dyed black (D), by the thermal camera.

The thermography instrumentation used to obtain thermal images of the seed samples from which the temperature measurement were obtained consisted of an IR camera and a laser with a time control. Thermal images of seeds were taken with the IR camera (i5; FLIR Systems Wilsonville, OR, USA) with lens $\mathrm{f}=6.8 \mathrm{~mm}$. The thermal images were obtained during the seed laser irradiation for one minute, every $3.75 \mathrm{~s}$. Also, thermal images were obtained after the laser was turned off, during $1 \mathrm{~min}$, each $3.75 \mathrm{~s}$. The power of the red laser source $(650 \mathrm{~nm})$ used in this study was $27.4 \mathrm{~mW}$. The energy during seed exposure was calculated according to the equation: Exposed energy in Joules = power $(\mathrm{mW}) \times$ time $(\mathrm{s})$, according Khalifa and Ghandoor (2011); E $=\mathrm{P} \times \mathrm{t}=1.64$ Joules. The distance between the laser and the maize seed samples was $0.13 \mathrm{~m}$. The seeds were placed in a fixed manner on a side opposite to the embryo and suspended in air (only supported side by a heat dissipater). 204 images for each condition of the seeds of each variety $\left(V_{1 C N}, V_{1 C D}, V_{2 F N}\right.$, and $\left.V_{2 F D}\right)$ were obtained ie a total of 816 thermal images. Temperature reading was taken from each thermal image at the point where the laser light impinges (the highest temperature value on the sample). The thermal radiation from the seed samples was measured under controlled conditions at a temperature $T_{0}=$ $T_{t=t}$, placing the instrumentation in a box made with insulating material.

The optical absorption spectra were obtained by photoacoustic spectroscopy (PAS), according to Hernandez et al. (2011). The studied seeds were randomly selected from each variety and each condition and were placed in the Photoacoustic (PA) cell. The optical absorption spectra were obtained in the wavelength range from 325 to $700 \mathrm{~nm}$. The cylindrical PA cell dimensions were 9 and $16 \mathrm{~mm}$ of height and diameter, respectively. The PA signal was pre-amplified before feed the input signal of the lock-in amplifier. The optical absorption coefficient $(\beta)$ was determined from 
T a b l e 1. Comparison of mean physical parameters of the studied varieties of maize seeds

\begin{tabular}{lclcccccc}
\hline Variety & $\begin{array}{c}\text { Productive } \\
\text { cycle }\end{array}$ & Description & $\begin{array}{c}\text { Length } \\
(\mathrm{mm})\end{array}$ & $\begin{array}{c}\text { Width } \\
(\mathrm{mm})\end{array}$ & $\begin{array}{c}\text { Thickness } \\
(\mathrm{mm})\end{array}$ & $a_{s} l_{s}$ & $\begin{array}{c}\mu \mathrm{s} \\
(\mathrm{mm})\end{array}$ & $\begin{array}{c}\tau \\
(\mathrm{ms})\end{array}$ \\
\hline 'Toluqueña' & 2012 & Crystalline & $12.08 \pm 0.38$ & $6.80 \pm 0.17$ & $3.78 \pm 0.21$ & 41.64 & $91 \times 10^{-3}$ & 41.14 \\
'Cacahuazintle' & 2012 & Floury & $10.05 \pm 0.20$ & $8.85 \pm 0.36$ & $4.13 \pm 0.18$ & 45.50 & $91 \times 10^{-3}$ & 33.90 \\
\hline
\end{tabular}

the PA signal amplitude using the equation proposed by Poulet et al. (1980) after verification that the sample was thermally thick ie $a_{s} l_{s}>>1$, with $a_{s}=(\pi f / \alpha)^{1 / 2}=\mu_{s}^{-1}$, where $\alpha$ and $l_{s}$ are the sample thermal diffusivity and thickness, respectively, and $\mu \mathrm{s}$ is the thermal diffusion length. $\alpha=4.44$ $\mathrm{x} 10^{-3} \mathrm{~cm}^{2} \mathrm{~s}^{-1}$ according to Hernandez et al. (2009) and $f=17 \mathrm{~Hz}$. Having verified the necessary condition for thermally thick samples $\left(a_{s} l>>1\right.$, Table 1$)$, the optical absorption coefficient value $(\beta)$ was calculated and from this value the optical penetration length $\left(l_{\beta}=1 / \beta\right)$ was obtained. The $l_{s}$ value for each sample corresponds to the average thickness of each genotype and condition. At a wavelength of 650 $\mathrm{nm}$, the PA signal amplitude and phase as a function of the light modulation frequency was obtained from 12 to $30 \mathrm{~Hz}$. From these data and using the thermal diffusion model of Rosencwaig and Gersho (R and G), it is possible to obtain the non-radiative relaxation time $(\tau)$, for the case of thermally thick and optically transparent samples (which is the case of the present study). In this case, the PA signal amplitude varies as $f^{-1}$ and the PA signal phase as a function of the light modulation frequency behaves according to Baesso et al. (1989), equation:

where: $\omega=2 \pi f$ is the angular frequency and $\tau_{\beta}=1 / \beta^{2} \alpha_{s}$ is the thermal diffusion time. The first term in the equation

$$
\varphi=\frac{3 \pi}{4}+\tan ^{-1}(\omega \tau)-\tan ^{-1}\left[\frac{1}{1+\left(2 \omega \tau_{\beta}\right)^{\frac{1}{2}}}\right],
$$

corresponds to the nonradiative relaxation contribution, whereas the second one is the contribution from the thermal diffusion within the optical absorption length.

\section{RESULTS}

Both varieties of maize: 'Cacahuazintle' and 'Toluqueña' were thermally thick; also, it is possible to observe that the physical characteristics of the studied varieties $\left(V_{1 C}\right.$ and $\left.V_{2 F}\right)$ showed statistically significant differences in their physical parameters (Table 1). The comparison of the means of temperature measurements for the crystalline and floury seeds under the natural colour and black-dyed conditions obtained by thermal camera was conducted in two phases: a) during exposure to the laser light and b) after the laser light off (at the temperature decay stage).

As can be seen (Table 2), for the seeds in their natural colour (undyed, $V_{1 C N}$ and $V_{2 F N}$ ), from time $t_{2}$ to time $t_{16}$ (during the seed exposure to laser light), it was possi- ble to observe significant statistical differences $(p \leq 0.05)$ between changes in temperature measured in the crystalline and floury seeds $\left(V_{1 C N}\right.$ and $\left.V_{2 F N}\right)$, (Table 2). The floury seeds $\left(V_{2 F N}\right)$ (row b) exhibited the highest variation in temperature when both seeds (crystalline and floury) in their natural colour are compared, having a variation with respect to their initial temperature of $0.75^{\circ} \mathrm{C}$. In the period of temperature decay (turning off the laser light), it was possible to observe that there were no statistically significant changes between the evaluated seeds (Table 2). It is possible to observe (Fig. 1a) the temperature measured as a function of time for the crystalline and floury seeds during the exposure to laser light with a laser turned on and after the laser light was turned off. The temperature was evaluated every $3.75 \mathrm{~s}\left(t_{0}, t_{1}, t_{2}, t_{3} \ldots \ldots . t_{16}\right)$ taking thermal imaging for both periods (turning on and off the laser light) and obtaining the temperature from these images. It is possible to observe that from the time $t_{1}$ the floury seed variety showed higher temperature compared with the crystalline seed variety during the laser light exposure (60 s). When the laser was off, it was possible to observe that the temperature decreased more rapidly in the floury seed variety than in the crystalline seed variety.

For the black-dyed seeds, there were statistically significant differences $(\mathrm{p} \leq 0.05)$ from time $t_{1}$ to time $t_{16}$ (during the exposure of the seeds to the laser light) in the temperatures measured by the infrared camera for the crystalline ('Toluqueña') and floury ('Cacahuazintle') seeds, (Table 2 continuation). It is possible observe that the 'Cacahuazintle' seed variety $\left(V_{2 F D}\right)$ had the highest temperature variation $(\Delta T)$ of $9.28^{\circ} \mathrm{C}$, with respect to the initial temperature $T_{0}$, attained at $60 \mathrm{~s}$ of exposure to laser light, where the temperature of the floury seed variety reached the value of $T_{t 16}=32.8^{\circ} \mathrm{C}$, having an increment $\Delta T$ of $40.34 \%$ with respect to its initial temperature $T_{0}$. On the other hand, the temperature changes obtained of the black dyed seed varieties, during the temperature decay, can be observed in Fig. 2b. For the black dyed seeds, there were statistically significant differences ( $\mathrm{p} \leq 0.05)$ from time $t_{1}$ to time $t_{16}$ (laser off) in the temperatures obtained for the crystalline ('Toluqueña') and floury ('Cacahuazintle') seeds. It is possible to observe that the obtained temperature of the 'Cacahuazintle' seed variety decays more rapidly when compared with the evolution of temperature of the 'Toluqueña' seed variety (Fig. 1b), from 


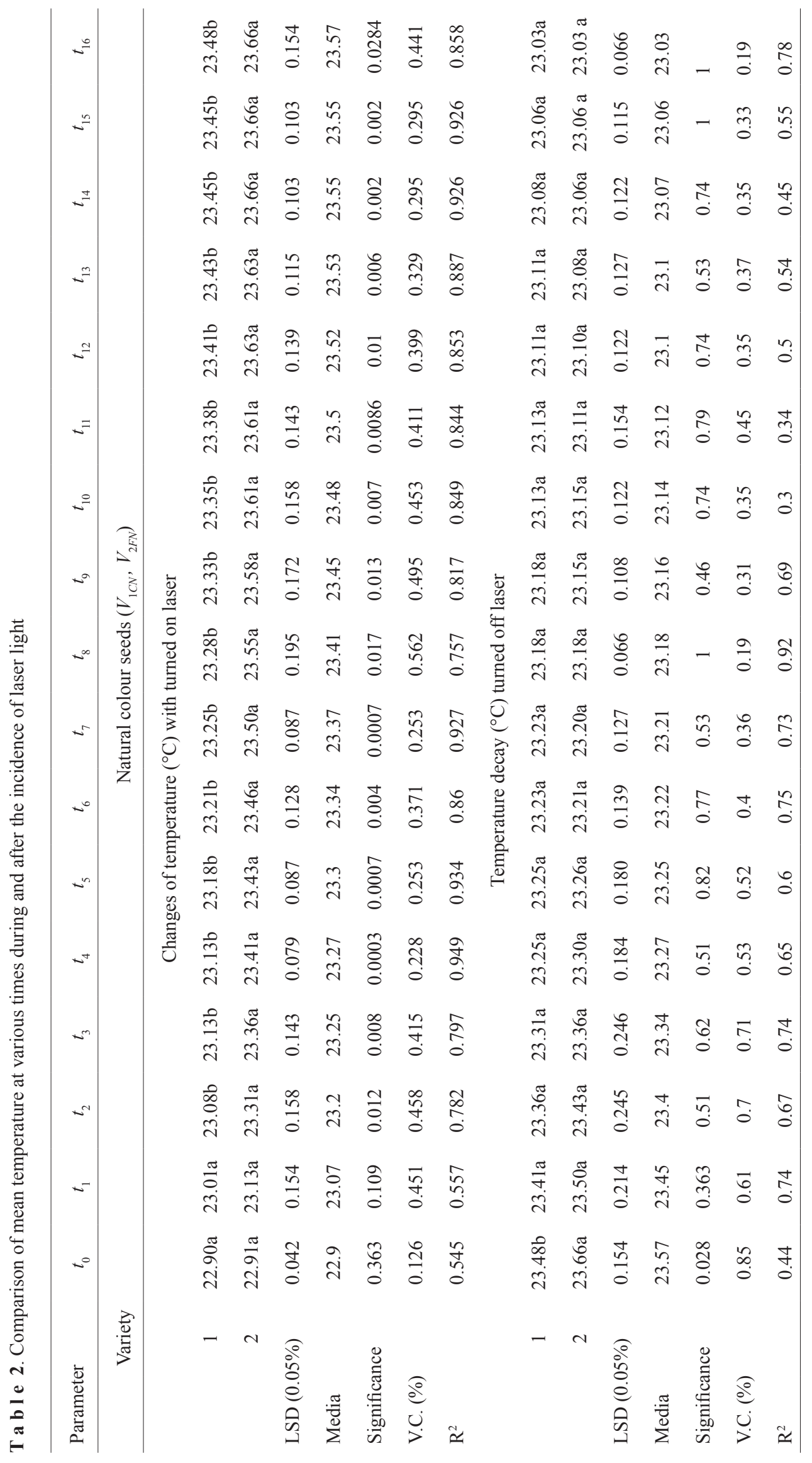




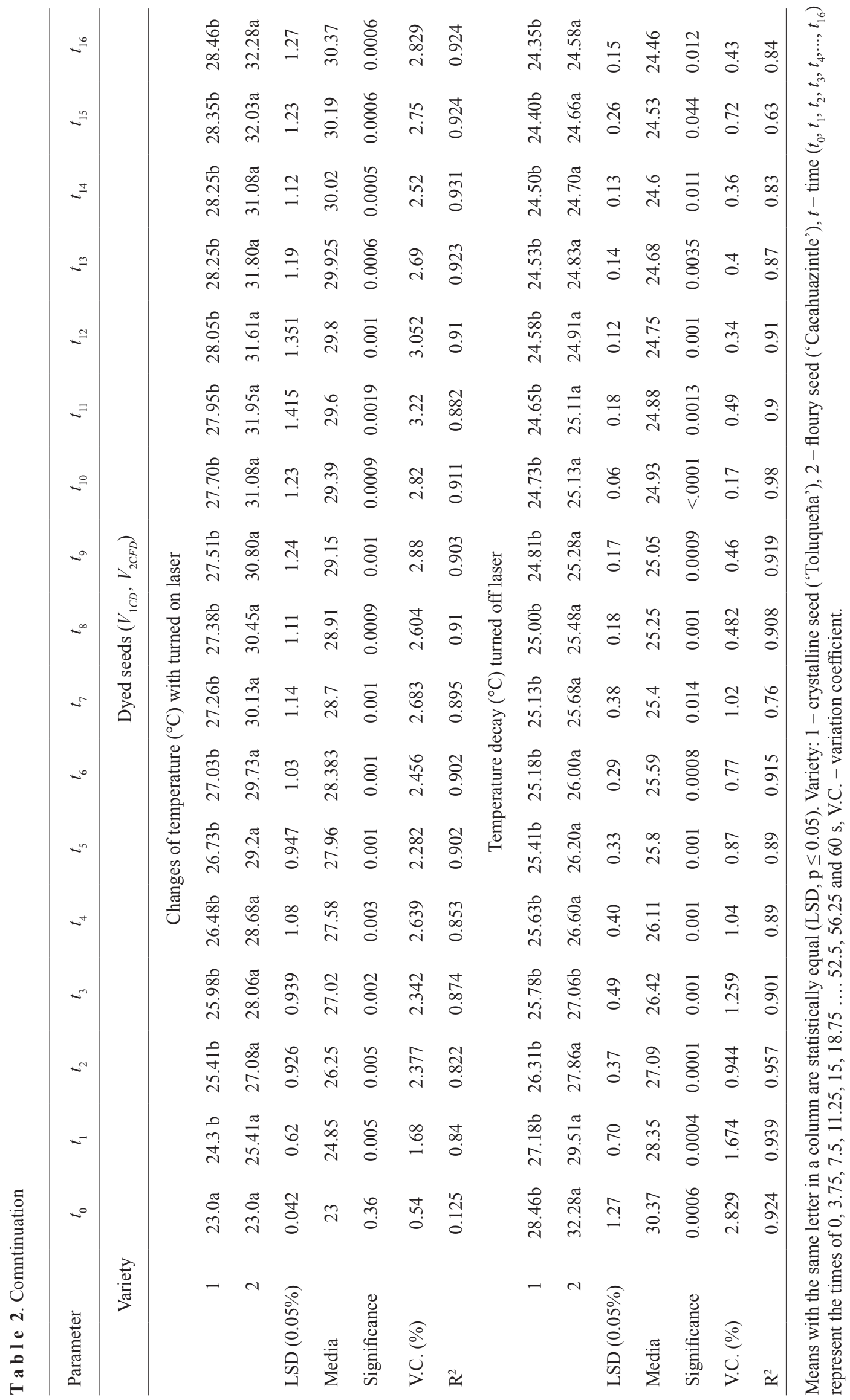


a

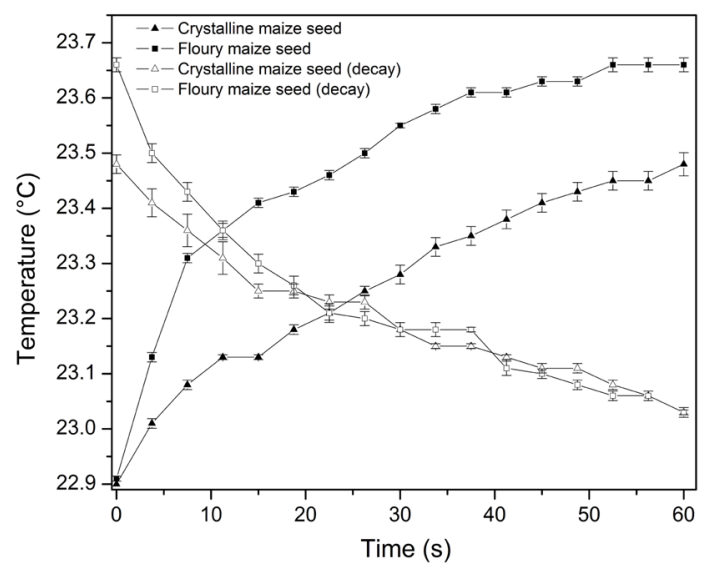

b

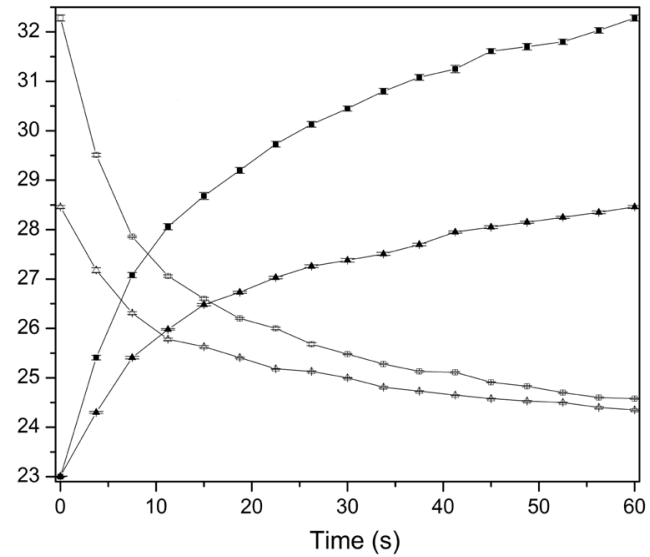

Fig. 1. Evolution and decay of temperature: a - maize seed in its natural colour (undyed) and b-dyed maize seeds (black dyed).

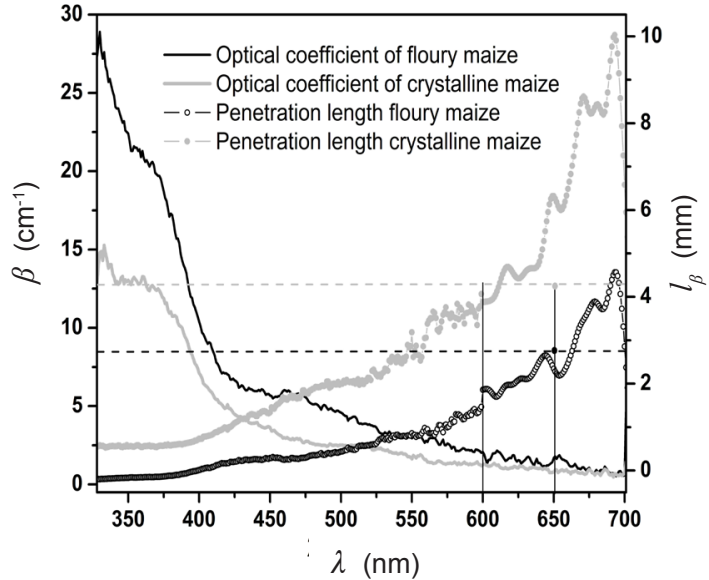

Fig. 2. Optical absorption coefficient $(\beta)$ and optical penetration length $\left(l_{\beta}\right)$ as a function of the wavelength for the $V_{1 C}$ and $V_{2 F}$ seed samples. The horizontal dashed lines represent the sample thickness $\left(l_{s}\right)$.

a

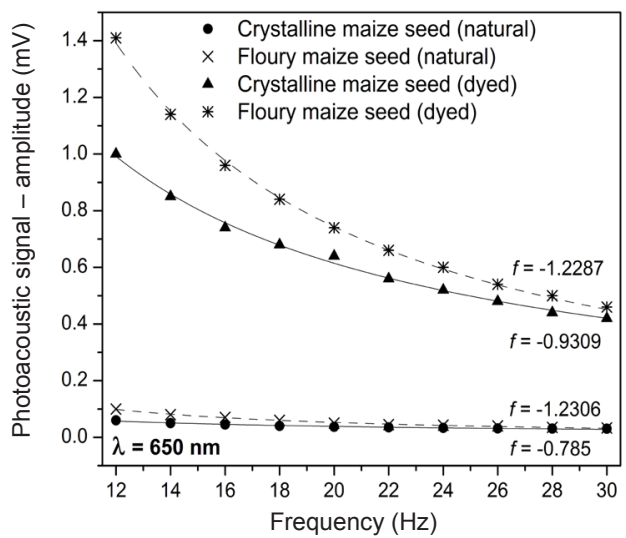

these dates it was observed that after $10 \mathrm{~s}$ the temperature of seeds 'Cacahuazintle' variety decreased by about $5^{\circ} \mathrm{C}$, and 'Toluqueña' variety by about $2.5^{\circ} \mathrm{C}$.

It is possible to observe the $\beta$ and $l_{\beta}$ obtained as a function of the wavelength (Fig. 2). The sample average thickness $\left(l_{s}\right)$ for each variety is denoted in this figure by horizontal dashed lines for both 'Toluqueña' $\left(l_{s C}\right)$ and 'Cacahuazintle' $\left(l_{s F}\right)$ seed varieties. It can be observed that $\beta$ decreases with the increasing wavelength, and conversely the $l_{\beta}$ increases with the increase of wavelength. It is possible to observe that maize seeds $V_{1 C}$ and $V_{2 F}$, at the $650 \mathrm{~nm}$ wavelength, are optically transparent and optically opaque, respectively, which means that for the case of the sample optically opaque $\left(l_{\beta}<l_{s}\right)$ (Fig. 2). Also, the thermal diffusion length $\left(\mu_{s}\right)$ is shorter than the optical penetration length in both cases $\left(V_{1 C}\right.$ and $\left.V_{2 F}\right)$, ie $\mu_{s}<l_{\beta}$ (Table 1). The $l_{\beta}$ obtained (right side axis) is shown as a function of wavelength. For the $V_{1 C}$ and $V_{2 F}$ seeds in the 325 to 615 and 325 to $670 \mathrm{~nm}$ ranges, the samples are optically opaque, respectively. For

$\mathrm{b}$

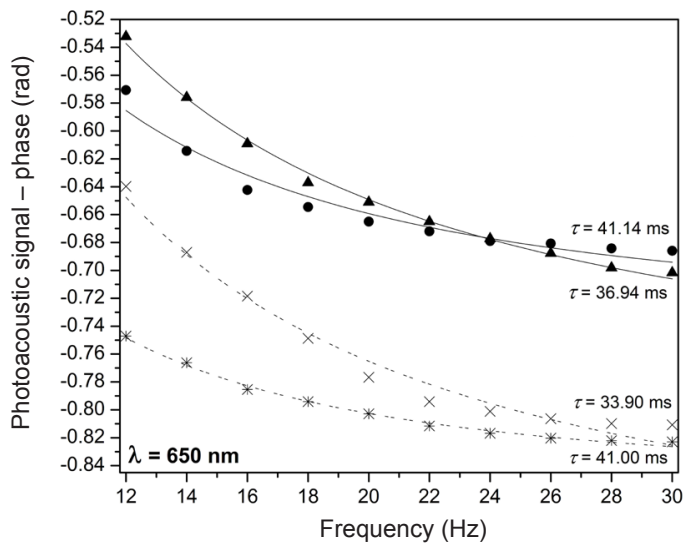

Fig. 3. a - Photoacoustic signal as function of frequency for crystalline and floury seeds in two conditions: natural and dyed, b - non radiative-relaxation times (thermal relaxation time) for crystalline and floury seeds: natural and dyed. 
wavelengths higher than 615 and $670 \mathrm{~nm}$, the seeds are optically transparent for the crystalline and floury seeds, respectively.

Figure 3 shows the behaviour of the amplitude and phase of the photoacoustic signal obtained by PAS at the $650 \mathrm{~nm}$ wavelength. Among the seeds evaluated, the floury black-dyed seed variety had a higher photoacoustic signal. From these data, the non-radiative relaxation time $(\tau)$ was obtained, which were 41.14 and $33.90 \mathrm{~ms}$ for the seeds without colouring for the crystalline and floury seed varieties, respectively. In the case of the dyed seeds, the $\tau$ values were 36.94 and $41 \mathrm{~ms}$ for the crystalline and floury varieties, respectively.

On the other hand, Fig. 4 shows the thermal images of the crystalline and floury maize seeds. There are eight columns; the first column refers to the seed optical images, the second column shows the thermal image before starting the irradiation process, the third, fourth, and fifth columns show thermal images during the irradiation process (at 15, 33.75, and $48.75 \mathrm{~s}$ ), and the sixth to the ninth columns show the thermal images after the irradiation process (at 7.5, 26.25, 45 , and $60 \mathrm{~s}$ ). The thermal image of the crystalline seeds (see row a) compared with the thermal images of the floury seeds (see row b) provides a difference due to differences in heating of the seeds by the laser light, being observed that the floury seeds show higher temperature with respect to the crystalline seeds. Also, the red and white colours denote the higher temperatures. Similarly, when the floury and crystalline dye seeds are compared (see rows c and d), the dyed floury seeds had a higher temperature change. The red and white colours mean that there was more heating in the place of LILI. These images imply that in both seeds in their natural and coloured condition, the floury seed variety had a higher temperature increment.

\section{DISCUSSION}

In the present investigation, we have studied the effects of low intensity laser irradiation applied during one minute on maize (Zea mays L.) seeds of two genotypes (crystalline and floury) evaluating the temperature changes measured by a thermal camera. From the results it can be said that, it is possible to observe temperature changes in crystalline and floury seeds with laser light at $650 \mathrm{~nm}$ and $27.4 \mathrm{~mW}$ and to quantify them using an infrared camera. For seeds in their natural state $\left(V_{1 C N}\right.$ and $\left.V_{2 F N}\right)$, the temperature changes were from 0.58 to $0.76^{\circ} \mathrm{C}$ for the crystalline and floury seeds, respectively, at a time of irradiation exposition $t_{16}$ $=60 \mathrm{~s}$. For the dyed seeds $\left(V_{1 C D}\right.$ and $\left.V_{2 F D}\right)$, the temperature changes were higher with respect to $V_{1 C N}$ and $V_{2 F N}$ and reached 5.56 and $9.28^{\circ} \mathrm{C}$ for the crystalline and floury seeds, respectively. The floury seed variety $\left(V_{2 F N}, V_{2 F D}\right)$ had a higher temperature change in the natural and dyed condition.

In this research, it was also found that among the seeds in their natural colour condition, the floury seed variety $\left(V_{2 F N}\right)$ had a higher $\beta$ value, lower optical penetration length $l_{\beta}$, at $\lambda=650 \mathrm{~nm}$, and lower $\tau$, compared with the crystalline seed variety $\left(V_{1 C N}\right)$. In the floury seed $l_{\beta}<l_{s}$ allowing that the incident laser light energy is absorbed into the seed. In the crystalline seed variety $V_{1 C N}, l_{\beta}>l_{s}$, so that the incident energy of the laser light, is absorbed within the crystalline seed in less proportion with respect to the absorbed for the

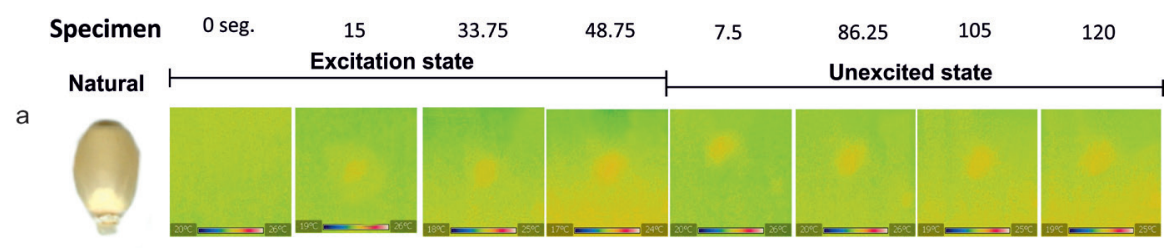

b

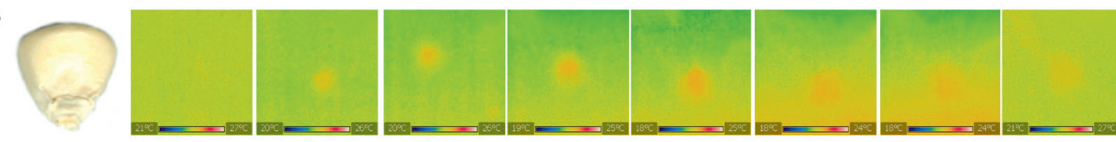

c Dyed

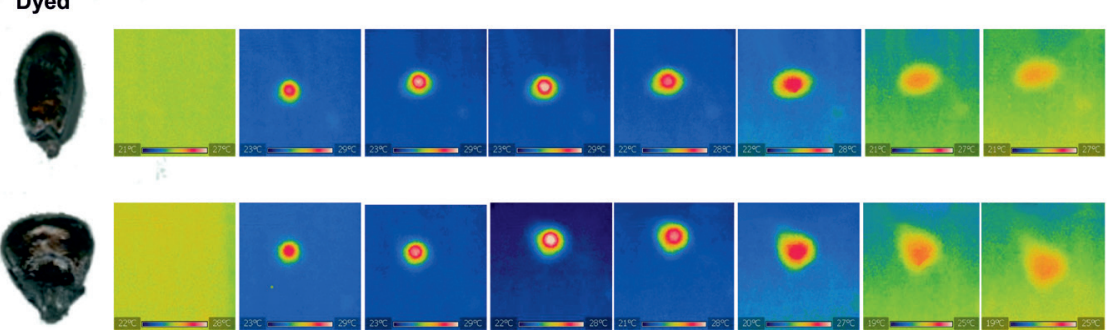

Fig. 4. Thermal images obtained with the thermal camera from the seeds: a - crystalline seed in its natural condition (undyed) ( $\left.V_{1 C N}\right)$, $\mathrm{b}$ - floury seed in its natural condition (undyed) $V_{2 F N}, \mathrm{c}$ - crystalline black-dyed seed $\left(V_{1 C D}\right)$, and d-floury black-dyed seed $\left(V_{2 F D}\right)$. 
floury seed. Moreover, the crystalline seed variety diffuses the heat better, because the molecules are better organized (less amorphous) (Da Silva et al., 1993) and the heat flows better; therefore, at the surface the temperature is lower. The floury seeds diffuse the heat into the seed with more difficulty; therefore, the increase in the temperature is higher at the surface. In this way, the increase in the temperature is higher at the surface of the floury seeds. This was reported by Da Silva et al. (1993), who found that the values of thermal diffusivity and conductivity are higher the in popcorn pericarp (crystalline seed) than in non-crystalline seeds.

Therefore, it has been demonstrated in this research using thermal images that crystalline and floury seeds diffuse the heat in different ways; and thus temperature changes produced by the laser light and received by the infrared camera are different. Where, higher temperature changes occurred when the crystalline and floury seeds were dyed black, in which case the temperature was increased by $9.28^{\circ} \mathrm{C}$ above the initial temperature of the floury seeds after one minute.

Photosensitizers, such as dyes, have been reported to enhance the laser effect as biostimulators (Ouf and AbdelHady, 1999; Hernandez et al., 2006; 2008; 2010). It was interesting to study the role of laser irradiation in the temperature changes of maize seeds in two conditions, natural and pretreated with a black dye, and to find that seed coloration modified the temperature changes in seeds exposed to the LILI.

Ouf and Abdel-Hady (1999) found that the effect of pretreating the soybean seeds with different dyes (methyl red, crystal violet, and methylene blue) caused a more pronounced effect of laser light on germination when compared with non-irradiated seeds. Also, when the laser was used to reduce the fungal contamination of the soybean seeds, particularly when the seeds were pretreated with dyes, the methylene blue was the most effective dye in enhancing the fungicidal effect of laser irradiation.

Other studies where seeds were photosensitized before laser irradiation have been reported. For example, pre-irradiation use of red methyl as a dye was found to increase significantly the seedling emergence rate, seedling dry weight, and field emergence, where the highest positive responses were found for the 30- and 60-s laser irradiations and laser intensities of 3.2 and $20 \mathrm{~mW} \mathrm{~cm}^{-2}$, respectively (Hernandez et al., 2006). Other studies on the same seeds with similar irradiation parameters reported minor effects on the variables of vigour (emergency, dry weight, and emergence velocity), when the seeds were irradiated in their natural colour (without dyed) (Hernandez et al., 2007).

The change of colour modifies seed light absorption as well as the temperature changes produced therein to be irradiated by low intensity laser light, as demonstrated in this investigation. Also in the present study, it has been found that changes in the seed colour increase the temperature changes, which are higher in the floury seeds, because they are less able to diffuse heat.
It could be said that the crystalline and floury seeds, given their optical and thermal properties, have a different way of absorbing the energy of the laser light and therefore increase their internal energy differently; consequently, the biochemical and physiological metabolic processes of seeds pre-treated with dyes could change their response to the applied laser light effect. Differences in light absorption and changes in temperature may be associated with the different effects produced by laser irradiation applied pre-sowing. Hernandez et al. (2009) reported positive, negative, and zero effects when applying the same parameters of laser irradiation in different seed genotypes. In the present investigation, it has been found that there is a thermal component associated with the mechanisms of laser biostimulation, which is a function of the seed characteristics and their chromophores as absorbing centres, which are modified to dye the seed with artificial colouring. Some authors mention that the local transient rise in temperature of absorbing biomolecules may cause structural changes and trigger biochemical activity such as activation or inhibition of enzymes (Rassam, 2010). Future research could be performed using different photosensitizers to increase the laser effects for biostimulation in agricultural seeds.

\section{CONCLUSIONS}

1. In this research, it has been found that 60 -s irradiation with laser light (at a $650 \mathrm{~nm}$ wavelength and $27.4 \mathrm{~mW}$ power) produces temperature changes in crystalline and floury seeds and these changes can be quantified by using an infrared camera. Comparing the mean temperature of the black dyed seeds (crystalline and floury) at various times during and after the incidence of laser light, it was found that there were statistically significant differences ( $\mathrm{p} \leq 0.05$ ) from time $t_{1}=3.75$ to time $t_{16}=60 \mathrm{~s}$. The floury seed variety had the highest temperature variation with respect to the initial temperature (during the irradiation laser exposition). In terms of the rate of temperature decay, it was found that the temperature of the crystalline seed variety decays more rapidly, compared with the evolution of temperature of the floury seed variety.

2. Comparing the means of temperature for crystalline and floury seeds under the natural colour condition obtained with thermal camera, it was found that from time $t_{2}=7.5$ to time $t_{16}=60 \mathrm{~s}$ (during seed exposure to laser light), there were significant statistical differences $(p \leq 0.05)$ between the changes in temperature measured in the crystalline and floury seeds. The floury seeds exhibited higher variation in temperature, when both seeds are compared. In the period of the temperature decay, no statistically significant changes were shown between the evaluated seeds.

3. It was also found that among the seeds in their natural colour condition, the floury seed variety had higher optical absorption coefficient value, lower optical penetration 
length, at $\lambda=650 \mathrm{~nm}$, higher photoacoustic signal, and lower non-radiative relaxation time, compared with the crystalline seed variety.

4. In the present investigation, it has been shown that there is a thermal component associated with the mechanisms of laser biostimulation, which is a function of the seed characteristics, their chromophores as absorbing centres, which are modified to dye the seed with artificial colouring. In this way, the effects of laser treatment on maize seeds involve at least a temperature effect. It is important to know the temperature changes in the seeds that have been irradiated with a laser beam because this could have substantial practical and theoretical importance.

\section{REFERENCES}

Abu-Elsaoud Abdelgaphafar M. and Tuleukhanov S.T., 2013. Can He-Ne laser induce changes in oxidative stress and antioxidant activities of wheat cultivars from Kasakhstan and Egypt? Sci. Int., 1, 39-50.

A El-Kereti M., A El-feky S., S Khater M., A Osman Y., and A El-sherbini E.S., 2013. $\mathrm{ZnO}$ nanofertilizer and $\mathrm{He}-\mathrm{Ne}$ laser irradiation for promoting growth and yield of sweet basil plant. Recent Patents Food, Nutrition Agric., 5, 169-181.

Aladjadjiyan A., 2012. Physical factors for plant growth stimulation improve food quality, food production-approaches, challenges and tasks. (Ed. A. Aladjadjiyan). Tech Publisher, Rijeka, Croatia.

Baesso M.L., Mansanares A.M., Da Silva E.C., Vargas H., and Miranda L.C., 1989. Phase-resolved photoacoustic spectroscopy and EPR investigation of $\mathrm{MnO} 2-$ and $\mathrm{CoO}$-doped soda-lime glasses. Physical review. B, Condensed matter, 40, 1880-1884.

Budagovskii A.V., Solovykh N.V., Budagovskaya O.N., Budagovskii I.A., Michtchenko A., and Hernandez V. M., 2012. Response of plant organisms to laser irradiation of different spectral composition. Russian Agric. Sci., 38, 367-370.

Chen Y.P., Liu Y.J., Wang X.L., Ren Z.Y., and Yue M., 2005a. Effect of microwave and He-Ne Laser on enzyme activity and biophoton emission of isatis indigotica fort. J. Integrative Plant Biol., 47, 849-855.

Chen Y.P., Yue M., Wang X.L., 2005b. Influence of He-Ne laser irradiation on seeds thermodynamic parameters and seedlings growth of Isatis indogotica. Plant Sci., 168, 601-606.

Da Silva W.J., Vidal B.C., Martins M.E.Q., Vargas H., Pereira A.C., Zerbetto M., and Miranda L.C., 1993. What makes popcorn pop? Nature, 362, 417-417.

Ferdosizadeh L., Sadat-Noori S.A., Zare N., and Saghafi S., 2013. Assessment laser pretreatments on germination and yield of wheat (Triticum aestivum L.) under salinity stress. World J. Agric. Res., 1, 5-9.

Gao L.M., Li Y.F., and Han R., 2014. He-Ne laser preillumination improves the resistance of tall fescue (Festuca arundinacea Schreb.) seedlings to high saline conditions. Protoplasma, 1-14.

Hernandez A.C., CarballoA.C., Artola A., and Michtchenko A., 2006. Laser irradiation effects on maize seed field performance. Seed Sci. Technol., 34, 193-197.
Hernandez A.C.,Carballo C.A., Michtchenko A., and Lopez B.J., 2007. Pre-treatment laser light on maize seed vigor. Int. E-journal Eng. Mathematics: Theory Appl., 1, 87-94.

Hernandez A.C., Cruz O.A., Ivanov R., Domínguez P.A., Carballo C.A., Moreno I., and Rico M.R., 2011. The optical absorption coefficient of maize seeds investigated by photoacoustic spectroscopy. Food Biophysics, 6, 481-486.

Hernandez A.C., Dominguez P A., Cruz O.A., Ivanov R., Carballo C.A., and Zepeda B.R., 2010. Laser in agriculture (Review). Int. Agrophys., 24, 407-422.

Hernandez A.C., Domínguez P.A., Cruz O.A., Ivanov R., Carballo C.A., Zepeda B.R., and Galindo S.L., 2009. Laser irradiation effects on field performance of maize seed genotypes. Int. Agrophys., 23, 327-332.

Hernandez A.C., Mezzalama M., Lozano N., Cruz O.A., Martinez E., Ivanov R., and Domínguez A.P., 2008. Optical absorption coefficient of laser irradiated wheat seeds determined by photoacoustic spectroscopy. Eur. Physical J. Special Topics, 153, 519-522.

Hoseini M., Mirshekari B., and Babazadeh-Igdir H., 2013. Influence of biophysical priming on seed germination and yield on two landraces of lemon-balm (Melissa officinalis L.). Notulae Scientia Biologicae, 5(2), 238-243.

Jamil Y., Perveen R., Ashraf M., Ali Q., Iqbal M., and Ahmad M.R., 2013. He-Ne laser-induced changes in germination, thermodynamic parameters, internal energy, enzyme activities and physiological attributes of wheat during germination and early growth. Laser Physics Letters, 10, 045606045614.

Javed N., Ashraf M., Aisha Akram N., and Al-Qurainy F., 2011. Alleviation of adverse effects of drought stress on growth and some potential physiological attributes in maize (Zea mays L.) by seed electromagnetic treatment. Photochem. Photobiol., 87, 1354-1362.

Jia Z. and Duan J., 2013. Protecting effect of He-Ne laser on winter wheat from UV-B radiation damage by analyzing proteomic changes in leaves. Advances Biosci. Biotechnol., 4, 823-829.

Joshi S., Joshi G.C., and Agrawal H.M., 2012. Study on the effect of laser irradiation on wheat (Triticum aestivum L.) variety PBW-373 seeds on zinc uptake by wheat plants. J. Radioanal. Nucl. Chem., 294, 391-394.

Khalifa N.S., and EI Ghandoor H., 2011. Investigate the effect of nd-yag laser beam on soybean (Glycine max) leaves at the protein level. Int. J. Biol., 3, 135-144.

Metwally S.A., Abou-Ellail M., Abo-Leila B.H., and Aboud K.A., 2013. Effect of laser radiation on the growth, anatomical and biochemical genetic markers of celosia argentea plants. Int. J. Academic Res., 5, 200-206.

Mohammadi S.K., Shekari F., Fotovat R., and Darudi A., 2012. Effect of laser priming on canola yield and its components under salt stress. Int. Agrophys., 26, 45-51.

Muthusamy A., Kudwa P.P., Prabhu V., Mahato K.K., Babu V.S., Rao M.R., Gopinath P.M., and Satyamoorthy K., 2012. Influence of Helium-Neon laser irradiation on seed germination in vitro and physico-biochemical characters in seedlings of Brinjal (Solanum melongena L.) var. Mattu Gulla. Photochem. Photobiol., 88, 1227-1235.

Ouf S.A. and Abdel-Hady N.F., 1999. Influence of He-Ne laser irradiation of soybean seeds on seed mycoflora, growth, nodulation, and resistance to Fusarium solani. Folia Microbiologica, 44, 388-396. 
Perveen R., Ali Q., Ashraf M., Al-Qurainy F., Jamil Y., and Raza Ahmad M., 2010. Effects of different doses of low power continuous wave He-Ne laser radiation on some seed thermodynamic and germination parameters, and potential enzymes involved in seed germination of sunflower (Helianthus annuus L.). Photochem. Photobiol., 86, 1050-1055.

Perveen R., Jamil Y., Ashraf M., Ali Q., Iqbal, M., and Ahmad M. R., 2011. He-Ne laser induced improvement in biochemical, physiological, growth and yield characteristics in sunflower (Helianthus annuus L.). Photoch. Photobiol., 87, 1453-1463.

Podleśny J., Stochmal A., Podleśna A., and Misiak L.E., 2012. Effect of laser light treatment on some biochemical and physiological processes in seeds and seedlings of white lupine and faba bean. Plant Growth Regulation, 67, 227-233.

Popov A.Y., Popova N.A., and Tyurin A.V., 2007. A physical model of the action of low-intensity laser radiation on biological objects. Optics Spectroscopy, 103, 671-677.
Poulet P., Chambron J., and Unterreiner R., 1980. Quantitative photoacoustic spectroscopy applied to thermally thick samples. J. Applied Physics, 51, 1738-1742.

Prośba-Białczyk U., Szajsner H., Grzyś E., Demczuk A., Sacala E., and Bąk K., 2011. Effect of seeds stimulation on sugar bee t productivity (in Polish). Listy Cukrovarnicke a Reparske, 127, 344-347.

Rassam Y.Z., 2010. The Effect of laser light on virulence factors and antibiotic susceptibility of locally isolated Pseudomonas aeruginosa. J. Appl. Sci. Res., 6, 1298-1302.

Srećković M., Vasić R., Dukić M., Jevtić S., and Jovanić P., 2014. The influence of diode and $\mathrm{He}-\mathrm{Ne}$ Lasers on corn and wheat seeds. J. Agricultural Sci. Technol., B, 4, 165-175.

Wu J., Gao X., and Zhang S., 2007. Effect of laser pretreatment on germination and membrane lipid peroxidation of Chinese pine seeds under drought stress. Frontiers Biology in China, 2, 314-317.

Zhang H., Zhang L., Tidemand-Lichtenberg P., Buchhave P., Xu X., and Li Y., 2011. Effect of laser and LED on enzymatic production of ceramide. Photochem. Photobiol., 87, 131-136. 